\title{
Tratamento cirúrgico de tumores malignos envolvendo ombro e membro superior: estudo de 10 casos
}

\author{
Surgical treatment of malignant tumors from the shoulder and upper limb: a \\ study of 10 cases
}

\author{
Leonardo José Vieira ${ }^{(1)}$; João Paulo Vieira ${ }^{(2)}$; Alexandre Ferreira Oliveira ${ }^{(3)}$; Rosyane Rena de Freitas ${ }^{(4)}$; Felipe Torres Rabêlo ${ }^{(5)}$; \\ Rafael Rabello Lista Mira ${ }^{(6)}$ e Jean Khoury José ${ }^{(7)}$
}

\section{Resumo}

Para alguns pacientes com neoplasias em região de ombro e/ou membro superior, a desarticulação interescapulotorácica (Cirurgia de Berger) ou a desarticulação escápulo-umeral, embora radicais, são o tratamento cirúrgico de escolha. No entanto, em casos selecionados, a escapulectomia ou a cirurgia de Tikhoff-Linberg (ressecção interescapulotorácica supra-umeral) pode ser uma alternativa. N este trabalho, avaliamos 10 pacientes submetidos às diversas cirurgias em região de ombro e membro superior, de 2000 a 2003, nos hospitais ASCO M CER, O ncológico e D r. João Felício, de Juiz de Fora (M G ), procedendo à discussão sobre a modalidade cirúrgica aplicada a cada caso, a relação entre tipo histológico, localização do tumor e/ou comprometimento de feixe vásculo-nervoso com a indicação de uma ou outra cirurgia, a recorrência local ou sistêmica relacionada à técnica cirúrgica, a utilização de terapias neoadjuvantes e adjuvantes à cirurgia, o tempo de acompanhamento clínico sem indício de recidiva e óbitos.

Palavras-chave: neoplasias; cirurgia; membro superior; ombro; desarticulação.

\begin{abstract}
For a few patients with tumors arising from the scapula or periscapular soft tissues, amputation of the forequarter, although radical, is the surgical treatment of choice. H owever, in selected cases, the scapulectomy or the TikhoffLinberg resection can be an alternative. We evaluated ten patients who underwent surgery in the shoulder and upper limb, from 2000 until 2003 at the hospitals ASCO M CER, O ncológico and D r. João Felício, in the city of Juiz de Fora, M G. We discuss the surgical technique, the relationship between tumor histology, site and/or vessels and nervous involvement, and the selection of one surgery or another, the local or systemic reccurrence related with the surgical technique, the use of neoadjuvants and adjuvants therapies with the surgery, the length of diseasefree follow-up, and death.
\end{abstract}

Key words: neoplasms; surgery; scapula; periscapular soft tissue; desarticulation.

Trabalho realizado no Hospital ASCOM CER- ASSOCIAÇÃO FEM ININA DE PREVENÇÃO E COMBATE AO CÂNCER DE JUIZ DE FORA (HOSPITAL MARIA JOSÉ BAETA REIS), Hospital O ncológico e H ospital Dr. João Felício - Juiz de Fora / M G.

${ }^{1}$ Especialista em Cirurgia O ncológica pela Sociedade Brasileira de Cancerologia (SBC), chefe do Serviço de Cirurgia do H ospital ASCOM CER de Juiz de Fora e professor de O ncologia da Faculdade de M edicina da Universidade Federal de Juiz de Fora (UFJF).

${ }^{2}$ Cirurgião do Tórax com pós-graduação em Cirurgia do Tórax pelo ITP - UFRJ (Instituto de Tisiologia e Pneumologia da U niversidade Federal do Rio de Janeiro), mestrado em Pneumologia pelo ITP - UFRJ, título de especialista em Cirurgia do Tórax pela Sociedade Brasileira de Cirurgia Torácica, professor de Cirurgia do Tórax da UFJF e professor de Cirurgia Ambulatorial da FAME (Faculdade de M edicina) de Barbacena / M G.

${ }^{3}$ Cirurgião O ncológico, titular do Colégio Brasileiro de Cirurgiões e professor substituto de Cirurgia Gastroenterológica da UFJF.

${ }^{4}$ Acadêmica da Faculdade de M edicina da UFJF.

${ }^{5}$ Acadêmico da Faculdade de M edicina da UFJF.

${ }^{6}$ Acadêmico da Faculdade de M edicina da U FJF.

${ }^{7}$ Acadêmico da Faculdade de M edicina da UFJF.

Endereço para correspondência: Leonardo José Vieira - H ospital ASCO M CER. Av Independência, 3500 - Bairro Cascatinha - Juiz de Fora - M G

- E-mail: ljvieira@terra.com.br 


\section{INTRODUÇÃO}

As alternativas cirúrgicas para os tumores envolvendo a região do ombro e membro superior incluem a amputação interescapulotorácica (cirurgia de Berger), a desarticulação escápulo-umeral, a ressecção interescapulotorácica supra-umeral (cirurgia de TikhoffLinberg) e a escapulectomia ${ }^{1,2}$.

1) Amputação Interescapulotorácica (C irurgia de Berger): compreende a ressecção de todo o membro superior e a cintura escapular, incluindo a escápula e uma porção da clavícula. As maiores indicações para este procedimento ablativo são grandes tumores intimamente ligados aos nervos principais ou ao suprimento vascular do membro superior. As contra indicações são as metástases à distância e os grandes envolvimentos da parede torácica. 3,4,5,6,7,8,9,10,11

2) D esarticulação Escápulo-U meral: cirurgia pouco comum para 0 tratamento dos tumores musculoesquel éticos. 0 casionalmente, uma fratura patológica na porção média do úmero, com um membro doloroso e sem função pode ser a indicação. Para os tumores primários, o déficit funcional dessa cirurgia é semelhante ao da amputação interescapulotorácica, porém freqüentemente as margens cirúrgicas são ineficientes. 2,11,12,13

\section{3) Ressecção Interescapulotorácica Supra-U meral} (C irurgia de Tikhoff-Linberg ): é uma opção cirúrgica conservadora para os tumores ósseos e de partes moles do úmero proximal e da cintura escapular. Consiste, em sua forma clássica, na ressecção do terço proximal do úmero, terço distal da clavícula e toda a escápula, associados às partes moles adjacentes. $\mathrm{N}$ a seleção dos pacientes não deve haver tumor em parede torácica e em feixe vásculo-nervoso. A função da mão e do antebraço após a cirurgia é parcialmente preservada, com a flexão do cotovelo e a estabilidade do ombro, obtida com ou sem a necessidade de órtese. 1,14,15,16,17

4) Escapulectomia: é indicada para o tratamento de tumores de partes moles localizados superficialmente à escápula e de tumores ósseos primários desta. A perda funcional é mínima após as ressecções parciais da escápula. A movimentação e a força do ombro são praticamente normais. As funções da mão e do cotovelo podem estar normais, porém dependem da extensão da ressecção e dos nervos remanescentes. As complicações são poucas e a reabilitação geralmente é completa. 18,19,20,21,22,23,24,25

A seleção cuidadosa dos pacientes a serem submetidos a cirurgias, sejam preservadoras ou radicais, é fundamental para prevenir a recidiva local e se obter melhores resultados.

\section{OBJETIVO}

O objetivo deste trabalho é a avaliação de 10 pacientes submetidos a tratamento cirúrgico de tumores malignos em região de ombro e membro superior, procedendo à discussão sobre a modalidade cirúrgica aplicada a cada caso, a relação entre o tipo histológico, a localização do tumor e/ou comprometimento de feixe vásculo-nervoso com a indicação de uma ou outra cirurgia, a recorrência local ou sistêmica relacionada à técnica cirúrgica, a utilização de terapias neoadjuvantes e adjuvantes à cirurgia, o tempo de acompanhamento clínico sem indício de recidiva e óbitos.

\section{METODOLOGIA}

A pesquisa constou da análise de prontuários do período de 2000 a 2003 de 10 pacientes submetidos à cirurgia em região de ombro e membro superior nos hospitais ASC O M CER, Dr. João Felício e O ncológico da Cidade de Juiz de Fora ( $M G$ ). Foram avaliados sexo, idade, localização do tumor, tipo histológico, cirurgia a qual o paciente foi submetido, emprego ou não de terapia neoadjuvante e/ou adjuvante, recorrência, óbito e sobrevida. Posteriormente, discutiu-se a escolha da modalidade cirúrgica aplicada a cada caso.

\section{RESULTADOS}

De um total de 10 pacientes, 6 eram do sexo masculino e 4, feminino. A variação de idade foi de 18 a 84 anos. Em quatro pacientes, o tumor acometia a escapula; em três, o braço; em um, braço e antebraço; e em dois, a região escápulo-umeral.

Com relação ao tipo histológico, quatro eram fibrossarcomas (alto grau); dois, sinoviossarcomas (bifásico); um, carcinoma basocelular; dois, condrossarcomas e um fibroistiocitoma.

D ois pacientes foram submetidos à radioterapia pré operatória, um à quimioterapia paliativa no pósoperatório tardio por metástase sistêmica e um à quimioterapia adjuvante à cirurgia.

As cirurgias realizadas foram: quatro desarticulações interescapulotorácicas (cirurgia de Berger); uma escápulo-umeral; três escapulectomias e duas ressecções interescapulotorácicas supra-umeral (cirurgia de TikhoffLinberg).

Em relação ao acompanhamento, dois encontramse sem evidências de recidiva em 6 meses; um em 8 meses; um em um ano e dois meses; um em um ano e quatro meses; um em dois anos e 10 meses; um em três anos e 9 meses. Um apresentou foco metastático 
pulmonar três meses após a cirurgia, estando em tratamento quimioterápico; dois foram ao óbito com o ocorrido no vigésimo quarto e no segundo dias de pósoperatório.

Foto 1: Peça cirúrgica da cirurgia de Berger.

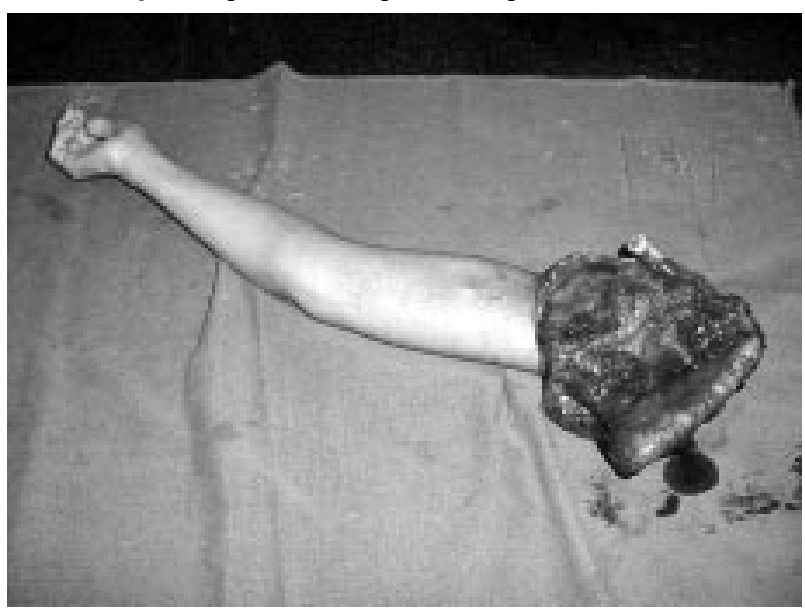

Foto 2: Intra-operatório da cirurgia de Tikhoff Linberg, evidenciando o isolamento do feixe vásculo-nervoso.

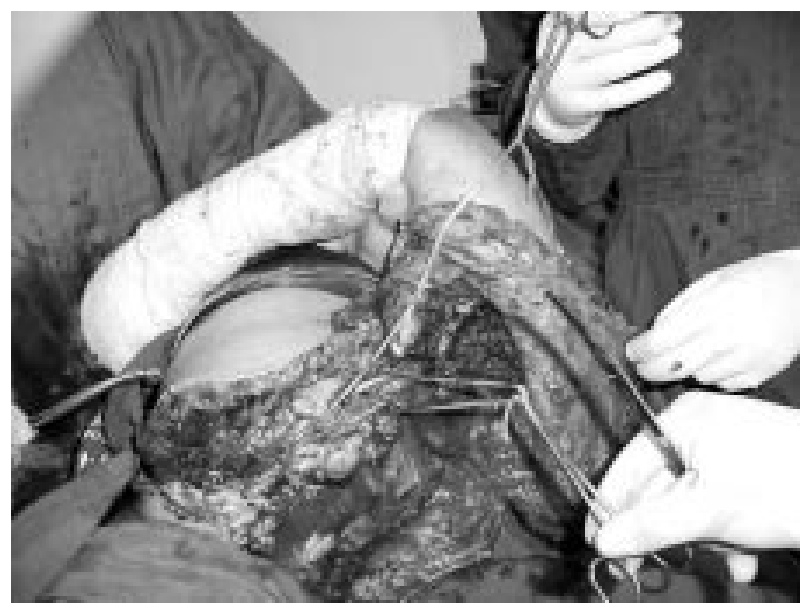

Foto 3: Paciente submetido à cirurgia de Berger - tumor acometendo braço proximal e antebraço.

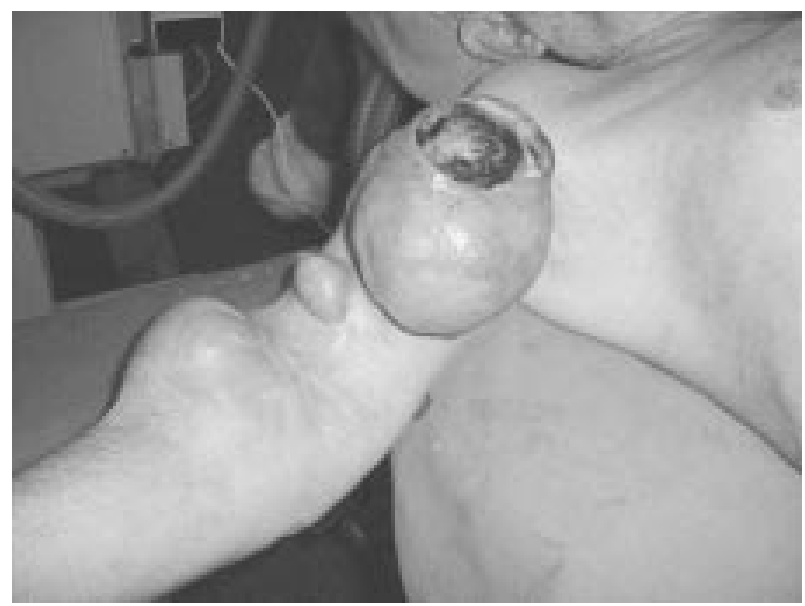

Foto 4: Tomografia computadorizada de paciente posteriormente submetido à cirurgia de Tikhoff Linberg, evidenciando comprometimento de clavícula, escápula, úmero proximal e partes moles adjacentes

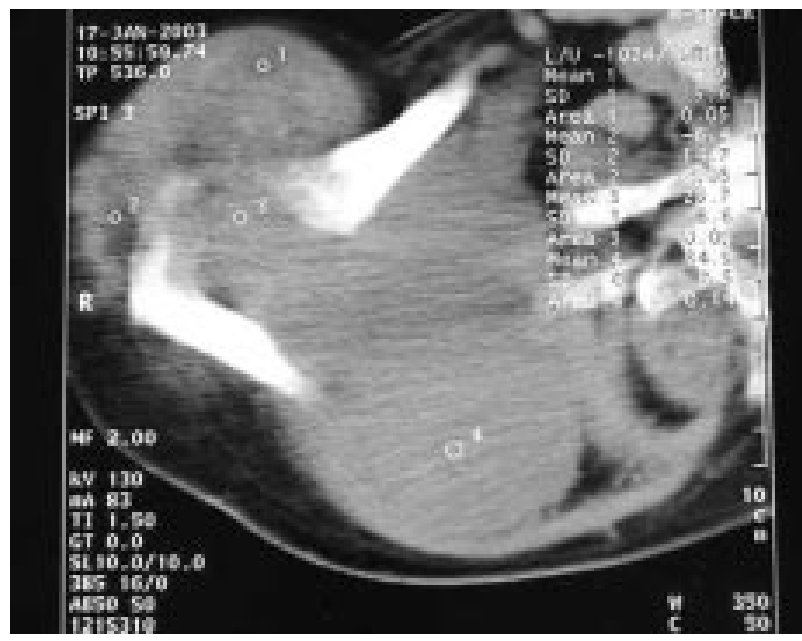

Gráfico 1: Com relação ao tipo histológico, 4 eram fibrossarcomas; 2, condrossarcomas; 2, sinoviossarcomas; 1, fibroistiocitoma e 1, carcinoma basocelular.

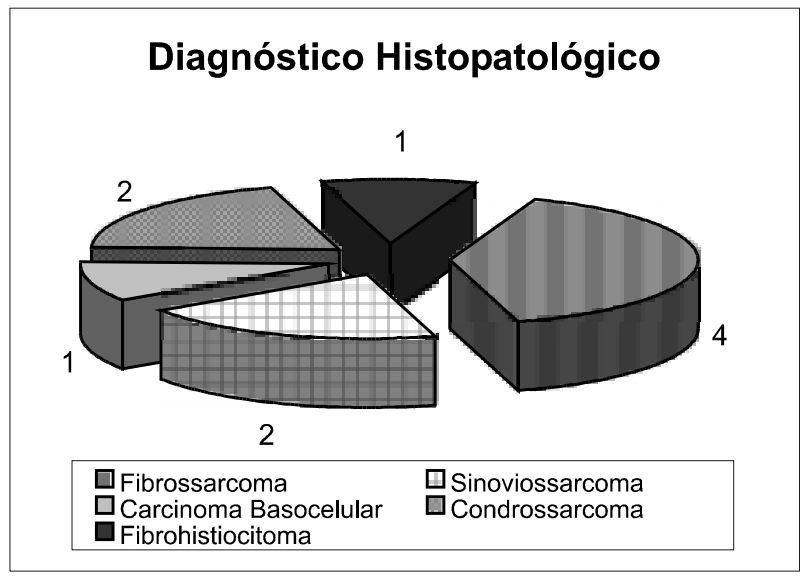

Gráfico 2: Com relação à localização do tumor, 4 casos comprometiam a escápula, 3 o braço, 2 a região escápulo-umeral e 1 o braço e antebraço.

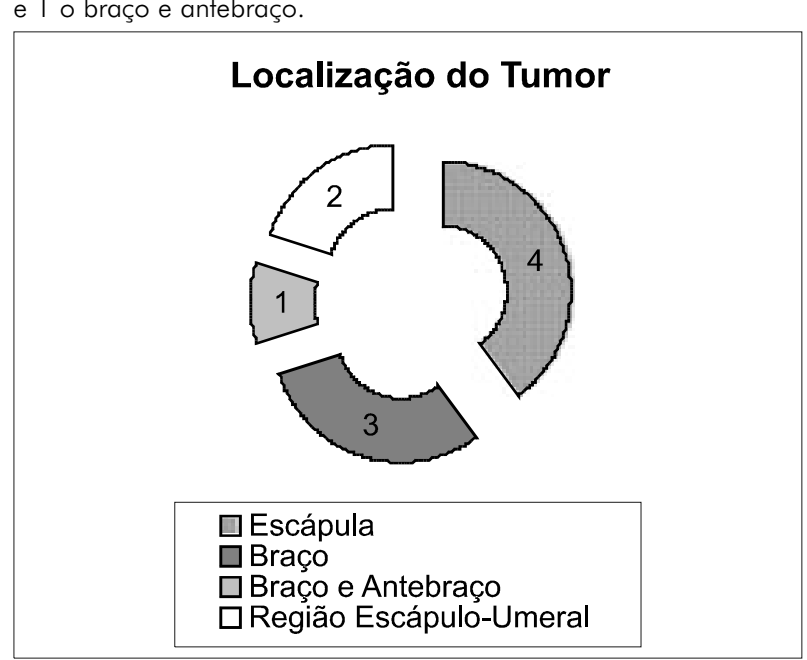


Gráfico 3: Com relação à cirurgia realizada, 4 foram cirurgia de Berger; 3, escapulectomia; 2, Tikhoff Linberg e 1, desarticulação escápulo-umeral.

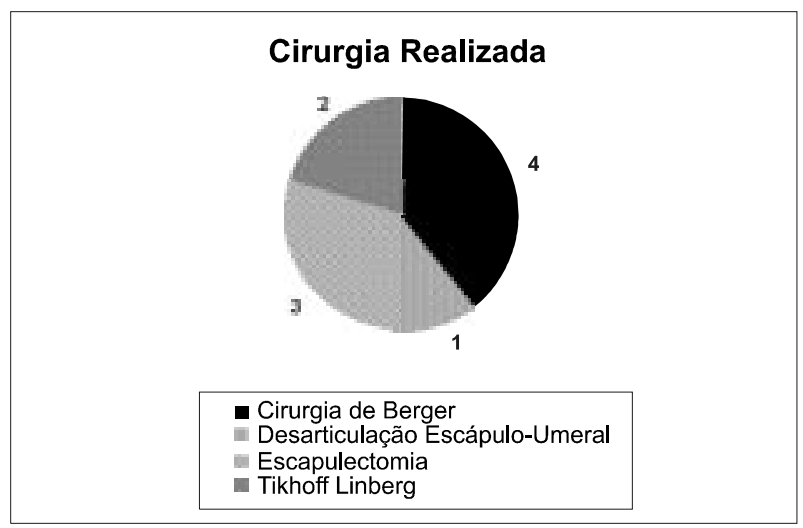

\section{DISCUSSÃO}

A cirurgia de Berger foi a cirurgia de escolha em quatro pacientes dos dez estudados, o que representa $40 \%$ da nossa amostra, contrariando Sugarbaker e $M$ alawer, que referem diminuição da indicação deste tipo de cirurgia atualmente ${ }^{8,11}$.

O s pacientes submetidos a esta modalidade cirúrgica tinham em comum um volumoso processo expansivo em topografia de braço e/ou cintura escapular com envolvimento de feixe vásculo-nervoso, impedindo cirurgias menos radicais.

Em uma paciente, a opção pela desarticulação escápulo-umeral deveu-se a tumor extenso, invadindo osso e feixe vásculo-nervoso em topografia de terço médio de braço, porém sem comprometimento da cintura escapular e com margens suficientes para garantir uma cirurgia oncológica adequada e evitar amputação mais radical.

Berger, em 1898 já mostrava uma incidência extremamente alta de recidiva nos casos submetidos à desarticulação escápulo-umeral devido a margens cirúrgicas comprometidas ou exíguas. 0 bservou ainda que o percentual de sobrevida diminuía sobremaneira se a cirurgia de Berger era levada a efeito como uma complementação em doente já submetido à desarticulação escápulo-umeral. ${ }^{3}$

Com conseqüências funcionais semelhantes entre a desarticulação escápulo-umeral e a cirurgia de Berger, a primeira leva a um resultado estético pouco melhor com menor dor fantasma?.

Bickels e colaboradores avaliaram 134 pacientes submetidos à cirurgia de Tikhoff-Linberg entre os anos de 1980 e 1997. D estes pacientes, 101 (75,4\%) obtiveram um resultado funcional bom ou excelente (movimentos do antebraço e da mão preservados em sua totalidadeno pós-operatório); $23,(17,1 \%$ ) moderado e $10,(7,5 \%)$ pobre ou ruim. ${ }^{1}$

Tivemos a oportunidade de indicar esta cirurgia em dois pacientes com resultados funcionais pós-operatórios satisfatórios - ressecção adequada do tumor e preservação dos movimentos do antebraço e da mão de forma fisiológica.

Alencar e Oliveira avaliaram cinco pacientes submetidos à escapulectomia por tumor ósseo maligno limitado à escápula. D estes, dois faleceram (um por metástase pulmonar e outro por condição não relacionada à doença), nenhum apresentou recidiva e todos mantiveram função normal do cotovelo e da mão, porém sem movimento ativo do ombro ${ }^{18}$.

Em nossa casuística a escapulectomia foi realizada com intuito curativo em dois casos, por tumor estritamente restrito à escápula. Como cirurgia paliativa, foi a opção para um caso objetivando melhoria da qualidade de vida, já que a paciente apresentava intensas dores locais sem resposta a narcóticos.

Karnofsky, ao avaliar 12 pacientes submetidos à cirurgia de Berger paliativa, obtevemelhoria da qualidade de vida em dois terços dos casos, com alívio da dor, promoção do bem estar físico na medida do possível e aumento de expectativa de vida com o mínimo de dignidade.27

A submissão de um paciente sabidamente fora de possibilidade terapêutica à cirurgia tem sua indicação devidamente estabelecida devido à dor intratável e/ou sangramento. ${ }^{13,26,27}$

C om relação à questão da recidiva, a literatura mostra que não há correlação entre as cirurgias conservadoras ou radicais se o estadiamento for corretamente estabelecido e se as margens cirúrgicas estiverem livres, ou seja, não se pode atribuir a recorrência local ou sistêmica a uma cirurgia preservadora se 0 anatomopatológico revelou margens cirúrgicas livres e se o estadiamento préoperatório não mostrou outras lesões e/ou comprometimento sistêmico. ${ }^{11}$

$\mathrm{N}$ os casos apresentados, recidiva local não foi observada. 0 único caso de recidiva nesta série ocorreu à distância, em paciente submetido à cirurgia conservadora de Tikhoff-Linberg que apresentou foco metastático pulmonar três meses após a cirurgia, com tomografia computadorizada do tórax normal no estadiamento préoperatório e diagnóstico do tumor primário de sinoviossarcoma .

Gentil em trabalho realizado no Hospital AC Camargo (SP) sobre tumores de partes moles mostra 0 fibrossarcoma apresentando tendência mais marcante à recidiva. $^{5}$ 
Nos casos de tumores com extremo comprometimento ósseo e de partes moles, a radioterapia pré-operatória é uma boa opção a fim de se reduzir a extensão da lesão para posterior abordagem cirúrgica ${ }^{12}$. D ois dos nossos pacientes se beneficiaram deste tratamento.

A quimioterapia tem sua indicação adjuvante nos casos de sarcoma de alto grau e/ou em pacientes com menos de 50 anos, ou como forma paliativa nos casos de metástase sistêmica evidente. ${ }^{12}$ Esta modalidade terapêutica foi necessária em três casos, por tratar-se de paciente jovem ou como alternativa paliativa devido à metástase pulmonar.

Os dois pacientes que evoluíram ao óbito foram submetidos a cirurgias paliativas, tendo o desfecho ocorrido no vigésimo quarto e no segundo dias de pósoperatório.

Embora as cirurgias radicais sejam ainda necessárias para pacientes com tumor localmente avançado envolvendo a região do ombro e membro superior, muitos podem ter uma boa perspectiva com procedimentos cirúrgicos conservadores voltados para 0 tratamento da doença, maximizando o resultado funcional ${ }^{11}$.

A literatura médica mostra que as amputações maiores da cintura escapular não aumentam a sobrevida se comparadas a cirurgias menos agressivas, sendo a escolha da abordagem operatória ligada à extensão da lesão e à sua relação com feixes neurovasculares. A realização de cirurgias radicais, como a desarticulação interescapulotorácica, por exemplo, é tecnicamente menos trabal hosa comparada à cirurgias preservadoras do tipo T ikhoff-Linberg e oferece resultados oncológicos equivalentes em tumores cuja localização permita a realização de ambas as técnicas, porém não se deve deixar de indicar uma cirurgia que possibilite melhor qualidade de vida ao paciente no pós-operatório com ressecção adequada do tumor por desconhecimento técnico, praticidade, ou qualquer outra razão ${ }^{11}$.

\section{REFERÊNCIAS BIBLIOGRÁFICAS}

1. BickelsJ, WittigJC, Kollender Y, Keller-Graney K, M eller I, M alawer M M . Limb-sparing resections of the shoulder girdle. J Am Coll Surg 2002;194-4.

2. Sugarbaker $P$. Alternativas cirúrgicas para as ressecções da cintura escapular. In: M alawer M M , Sugarbaker PH , Lopes A. Atlas de cirurgia para sarcomas ósseose departesmoles. 1a ed. São Paulo: Lemar; 2003. p.77-85.

3. Berger P. L'amputation interescapulo-thoracique dans le treatment des tumeurs malignes del'extrémitésupérieure del'humérus. Rev Chir 1898;18:861-85.
4. Fanous N, Didolkar M S, H olyokelD. Evaluation of forequarter amputation in malignant diseases. Surg $\mathrm{G}$ ynecol O bst 1976;142:381-4.

5. G entil FC. Indicações, técnica, tática cirúrgica eresultados da amputação inter-escápulo-torácica no tratamento das neoplasias malignas [tese]. C ampinas (SP): Faculdade de Ciências M édicas da PontifíciaU niversidadeC atólica; 1978.

6. Gläser A, Schauer K. Interscapulothoracic amputation, desarticulation of thehip, hemipel vectomy: indicationsand results. Zentralbl Chir 1979;104(18):1221-4.

7. Sim FH, Pritchard DJ, I vins JC. Forequarter amputation. O rthop Clin N Am 1977;8:921-31.

8. Sugarbaker P, M alawer M . Amputação interescapulotorácica. In: $M$ alawer M M , Sugarbaker PH , LopesA. Atlasdecirurgia para sarcomas ósseos e de partes moles. 1a ed. São Paulo: Lemar; 2003. p.145-54.

9. LevineEA, Warso M A, M cC oy D M , D asGuptaTK. Fore quarter amputation for soft-tissuetumors. Am Surg 1994; 60:367-70.

10. Bhagia SM , Elck EM , G rimer RJ. Forequarter amputation for high-grademalignant tumors of the shoulder girdle. J Bone Joint Surg 1997;79:924-6.

11. Clark M A, Thomas J M . M ajor amputation for soft-tissue sarcoma. BrJ Surg 2003;90(1):102-7.

12. Karakousis CP. Sarcomas of the soft tissues. In: Bland KI, D alyJM , Karakousis CP. Surgical oncology: contemporary principles\& practice. 1a ed. N ew York: M cG raw-H ill. p. 983-1014.

13. Clark M A, Thomas JM . Amputation for soft-tissue sarcoma. Lancet O ncol 2003;4(6):335-42.

14. $\mathrm{H}$ ahn SB, Kim N H , Choi N H . Treatment of bonetumors around the shoulder joint by theT ikhoff-Linberg. Yonsei M ed J 1990;31(2):110-22.

15. H am SJ, H oeskstraH J, EismaW H, Schraffordt KoopsH, O Idhoff J. The Tikhoff-Linberg producere in the treatment of sarcomas of theshoulder girdle. J Surg 0 ncol 1993; 53(2):71-7.

16. Sugarbaker $P, M$ alawer M . Cirurgia deT ikhoff-Linberg e suas modificações. In: M al awer M M , Sugarbaker PH , Lopes A. Atlas de cirurgia para sarcomas ósseos edepartes moles. 1a ed. São Paulo: Lemar; 2003. p. 373-88.

17. Voggenreiter $G$, Assenmacher $S$, Schmit-N euerburg KP. Tikhoff-Linberg producerefor boneand soft tissuetumors of the shoulder girdle. Arch Surg 1999;134(3):252-7.

18. Alencar PGC, O liveiraEC. Escapulectomia no tratamento dos tumores ósseos malignos primários. Rev Bras 0 rtop 1994;29(11/12):791-4.

19. EstapéG , C resseri A, Scigliano H , I raola M I, D elgado B. Subtotal resection of thescapuladueto a chondrosarcoma. 
Cir U rug 1998;58(1):51-5.

20. G uptaT. Escapulectomia: ressecção da cintura escapular do tipo III. In: M alawer M M , Sugarbaker PH , Lopes A. Atlas decirurgia para sarcomas ósseos e de partes moles. 1a ed. São Paulo: Lemar; 2003. p. 389-98.

21. Vasconcelos N eto AS, Vasconcelos GR, Valenca M A. Escapulectomia: resultado funcional: relato de caso. Rev Bras 0 rtop 1984;19(2):53-6.

22. Volpe CM, Pele M, D oerr RJ, Karakousis CP. Radical scapulectomy with limb salvage for shoulder girdle soft tissue sarcoma. Surg 0 ncol 1996;5(1):43-8.

23. Gibbons CL, Bell RS, Wunder JS, G riffin AM , 0 'Sullivan $B, C$ atton $C N$, et al. Function after subtotal scapulectomy for neoplasm of boneand soft tissue. J BoneJ oint Surg Br 1998;80(1):38-42.
24. M arkhede G, M onastyrski J, Stener B. Scapulectomy for malignant tumor function and shoulder strength in five patient. Acta O rthop Scand 1985;56:332-6.

25. Papaioannou AA, Francis KC. Scapulectomy for the treatment of primary malignant tumors of the scapula. Clin Orthop 1965;135:165-70.

26. WitigJC, Bickels J, Kollender Y, K ellar-G raney KL, M eller I, M alawer M M . Palliativeforequarter amputation for metastatic carcinoma to the shoulder girdle region: indications, preoperativeevaluation, surgical technique, and results. J Surg O ncol 2001;77(2):105-13.

27. M erimsky 0 , Kollender $Y$, Inbar M , Lev-CheloucheD, G utman M , Issakov J, et al. Is forequarter amputation justified for palliation of intractable cancer symptoms? Oncology 2001;60(1):55-9. 